\title{
Eigenspannungsorientiertes Fügen von hybriden Bauteilen mittels Radial-Walzen
}

\author{
Christina Guilleaume ${ }^{1}$ (D) - David Kühne ${ }^{2}$ Markus Kästner ${ }^{2}$ - Alexander Brosius
}

Eingegangen: 17. Januar 2021 / Angenommen: 9. April 2021 / Online publiziert: 28. April 2021

(c) Der/die Autor(en) 2021

\section{Zusammenfassung}

Flexible Fertigungsprozesse in der Umformtechnik ermöglichen es, schnell und effizient auf veränderliche Rahmenbedingungen beispielsweise durch Kundenwünsche und rechtliche Anforderungen reagieren zu können. Besonders die inkrementellen Umformverfahren der Massivumformung zeichnen sich durch eine hohe Flexibilität aus, da die Prozesse gut anpassbar sind und die verwendeten Anlagen und Werkzeuge in einem geringeren Maße auf ein spezifisches Bauteil hin ausgelegt werden.

Der hier vorgestellte Fügeprozess mittels Radial-Walzen ist geeignet, hybride Bauteile form- und kraftschlüssig miteinander zu verbinden. So lassen sich die spezifischen Vorteile verschiedener Werkstoffe kombinieren und Nutzungs- sowie Leichtbaupotenziale können gezielt genutzt werden. Die in diesem Prozess entstehende charakteristische umlaufende Kerbe in der Wellenkomponente bildet eine Schwachstelle der gefügten Bauteile, da es dort unter Last zu einer Spannungskonzentration kommt, welche die Bildung eines Risses begünstigt. Dieser Herausforderung wird mit einer Kombination der Ausnutzung der Kaltverfestigung des gewählten Werkstoffs (1.7225) und gezielt eingebrachter Druckeigenspannungen in der Kerbe begegnet. Hier liegt der Fokus auf den Druckeigenspannungen, die das Potenzial aufweisen, sich verlängernd auf die Lebensdauer auszuwirken. Sie lassen sich gezielt über eine günstige Wahl des Walzpfads zur Herstellung der Fügeverbindung beeinflussen, wie sowohl in numerischen Analysen als auch durch Eigenspannungsmessungen an Realbauteilen gezeigt wird. Es können sehr hohe Eigenspannungen in der kritischen Zone des Kerbgrunds eingebracht werden, die einen deutlichen Einfluss auf die Lebensdauer haben, wie auch die Lebensdauerberechnungen mittels des Örtlichen Konzepts deutlich zeigen.

\section{Residual stress oriented joining of hybrid components by radial-rolling}

\section{Abstract}

Flexible manufacturing processes in forming technology make a quick and efficient response to changing conditions, for example due to customer demands and legal requirements, possible. The incremental forming processes in the area of bulk metal forming in particular are characterized by a high degree of flexibility, as the processes are readily adaptable and the equipment and tools used are less component specific.

Christina Guilleaume

christina.guilleaume@tu-dresden.de

1 Professur für Formgebende Fertigungsverfahren, TU Dresden, 01062 Dresden, Deutschland
2 Institut für Festkörpermechanik, TU Dresden, 01062 Dresden, Deutschland 
The joining process presented here by means of radial rolling is suitable for joining hybrid components by a combined form-fit and force-fit. In this way, the specific advantages of different materials can be combined and application as well as lightweight potentials can be taken advantage of in a targeted manner. The characteristic circumferential notch in the shaft component created in this process forms a weak point in the joined components, as stress concentrations occur in the notch ground under load. This increases the risk of crack formation in this area. This challenge is met by a combination of exploiting the work hardening of the selected material (1.7225) and selectively inducing residual compressive stresses in the notch. The focus here is on the residual compressive stresses, which have the potential to extend the component's fatigue life. They can be specifically influenced by an advantageous choice of the rolling path for producing the joint, as shown both in numerical analyses and by residual stress measurements on real components. Very high residual stresses can be introduced in the critical zone of the notch ground, which have a significant influence on the service life, as is also evident in the fatigue life simulations using the local concept.

\section{Einleitung}

Flexible Fertigungsprozesse in der Umformtechnik bilden die Grundlage, um schnell und effizient auf veränderliche Anforderungen an Produkte und Halbzeuge, die sich aus veränderten Kundenwünschen, rechtlichen Rahmenbedingungen und neuen technischen Anforderungen ergeben, reagieren zu können [1]. Besonders die inkrementellen Umformverfahren der Massivumformung wie das Freiformschmieden, Rundkneten und auch das Ringwalzen zeichnen sich durch eine hohe Flexibilität aus, da die eingesetzten Werkzeuge und Anlagen weniger auf ein spezielles Bauteil hin ausgerichtet sind und zur Fertigung einer recht großen Bandbreite von beispielsweise Bauteilgrößen eingesetzt werden können [2]. Die umformtechnischen Fügeverfahren bilden eine Untergruppe der inkrementellen Umformverfahren und sind geeignet, ohne den Einsatz von Hilfswerkstoffen, dauerhafte Fügeverbindungen auch hybrider Komponenten zu erzeugen. Eine umfassende Übersicht über umformtechnische Fügeverfahren haben Mori et al. [3] zusammengestellt. Das hier näher vorgestellte Fügen mittels Radial-Walzen ordnet sich in die Gruppe der umformtechnischen Fügeverfahren ein und nutzt gezielt Eigenspannungen, um eine hohe Lebensdauer zu gewährleisten.

\section{Stand der Technik}

Die Erzeugung von Eigenspannungen ist bei der umformtechnischen Herstellung von Halbzeugen und Bauteilen unvermeidlich. Diese können in der Größenordnung der Fließgrenze liegen [4]. Da die Eigenspannungen einen Unsicherheitsfaktor bei der Bauteilauslegung und im späteren Einsatz darstellen, war bisher häufig das Ziel, ihren Einfluss gering zu halten und sie beispielsweise durch nachgelagerte Wärmebehandlungen weitgehend aus einem Bauteil zu entfernen. Zudem führen fehlende Informationen zu Eigenspannungen häufig zu einer Überdimensionierung von Komponenten, um einen vorzeitigen Ausfall in jedem Fall zu vermeiden [4]. Derzeit findet diesbezüglich ein gene- relles Umdenken statt und die gezielte Beeinflussung und sogar Nutzung von Eigenspannungen in der Fertigungstechnik rückt in den Fokus wie von Volk et al. übersichtsweise dargelegt [5]. Hierbei existieren sowohl Prozesse, die geeignet sind, die Eigenschaften dünnwandiger Bauteile $\mathrm{zu}$ verbessern als auch wie im hier vorliegenden Fall die von dickwandigen Komponenten. Also Beispiele für den gezielten Einsatz von Eigenspannungen in Blechbauteilen sei beispielsweise die Fertigung bistabiler metallischer geschlossener Rohrprofile [6] genannt oder die Einbringung von Eigenspannung in Elektroblechen zur Steigerung der Energieeffizienz über eine Beeinflussung der magnetischen Eigenschaften [7]. Auch die Rückfederung bei Blechumformoperation kann über geeignet eingebrachte Eigenspannungen beeinflusst werden [8].

Eine Übersicht von Strategien zur gezielten Anpassung von Eigenspannungen in der Massivumformung ist in [9] zu finden. Als mögliche Stellgrößen zur gezielten Beeinflussung werden die Optimierung der Werkzeuggeometrie, eine Veränderung des Prozessablaufs, die Kontrolle der Temperatur, die Umformgeschwindigkeit und die Stufenfolge bei Umformprozessen genannt. Im hier vorgestellten Prozess des Radial-Walzens wurde bisher primär die Prozessführung als Stellgröße genutzt, aber auch die Optimierung der Werkzeuggeometrie ist prinzipiell möglich.

Im Hinblick auf Ermüdungsfestigkeit bei zyklischer Belastung werden vor allem Eigenspannungen auf der Makroebene, d.h. 1. Art, als relevant angenommen [10] und daher nachfolgend näher betrachtet. Die Berücksichtigung von Eigenspannungen in Kaltumformverfahren, insbesondere mit dem Ziel einer gezielten Nutzung, um eine Verbesserung der Lebensdauer zu erreichen, ist derzeit noch nicht industrieller Stand der Technik. Um hier Fortschritte zu erzielen, ist ein grundlegendes Verständnis der Entstehung von Eigenspannungen im betrachteten Umformprozess und die Untersuchung ihrer Stabilität während der Belastung erforderlich. Untersuchungen hinsichtlich der Stabilität von Eigenspannungen unter Biegebeanspruchung deuten darauf hin, dass auch rein elastische Deformationen zu einer deutlichen Veränderung des Eigenspannungsprofils führen kön- 
Abb. 1 a Schematischer Aufbau der Walzanlage mit eingelegtem Bauteil; b Bauteile vor und nach dem Fügeprozess

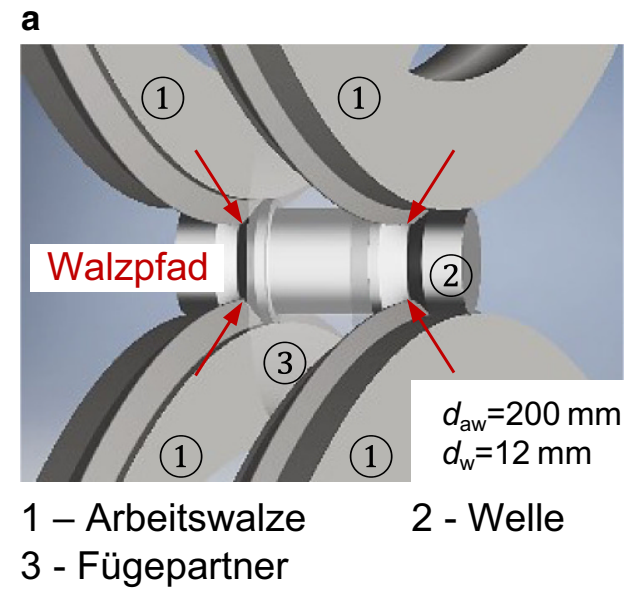

b

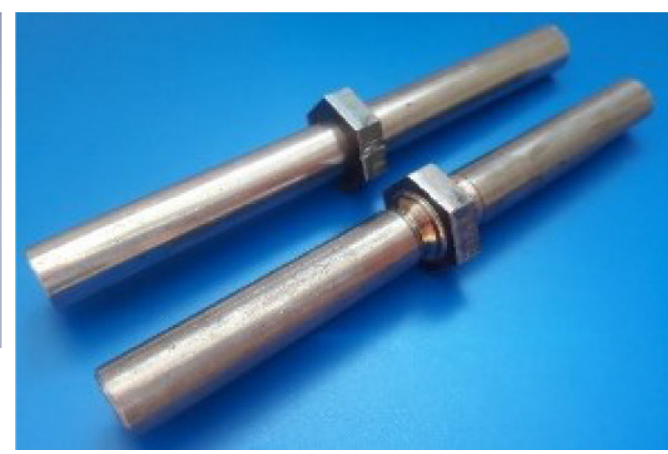

nen und die Lastrichtung der zyklischen Tests in Relation zur vorherrschenden Eigenspannungsrichtung von Bedeutung ist [11].

Grundsätzlich geht man davon aus, dass Druckeigenspannungen einen positiven Einfluss auf die Zug- und Ermüdungsfestigkeit von Bauteilen aufweisen, wohingegen Zugeigenspannungen einen negativen Einfluss haben [12]. Bei komplexeren Lastfällen ist eine solche Vereinfachung jedoch schwierig, da hier in der Regel mehrachsige Eigenspannungen mit komplexen Spannungszuständen infolge der Belastung überlagert werden, sodass eine einfache Beurteilung nicht möglich ist. Prozesse wie das Festwalzen und Kugelstrahlen, welche insbesondere bei Wellen aus dem Getriebebau regelmäßig zum Einsatz kommen, nutzen die positive Wirkung der eingebrachten Druckeigenspannungen in oberflächennahen Bereichen [13]. Beim Festwalzen überlagert sich die Wirkung der eingebrachten Druckeigenspannungen mit der im gleichen Schritt deutlich verringerten Oberflächenrauheit und einer Verfestigung der Randschicht durch den Umformprozess [14]. Die so eingebrachten Eigenspannungen können nahe der aktuellen Fließgrenze liegen und damit gerade bei stark kaltverfestigenden Stählen sehr hohe absolute Werte annehmen.

\section{Fügen durch Radial-Walzen}

Im hier vorgestellten Fügeprozess wirken zwei Paare von Arbeitswalzen (1) (Abb. 1), die in radialer und axialer Richtung zugestellt werden können. Dabei dienen jeweils die gegenüberliegenden Walzen gleichzeitig als Gegenlager für die auftretenden Prozesskräfte, siehe auch Abb. 1. Das zu walzende Bauteil (2) ist nicht eingespannt, sondern liegt frei auf einem zwischen den Walzen befindlichen Lineal auf. Die Arbeitswalzen mit dem Durchmesser $d_{\mathrm{aw}}=200 \mathrm{~mm}$ sind angetrieben, während das Bauteil mit dem Durchmesser $d_{\mathrm{w}}=12 \mathrm{~mm}$ nur aufgrund der Reibung an den Kontaktstellen in Rotation versetzt wird. Durch gezielte Wahl des Walzpfa- des, also der Kombination aus radialer und axialer Zustellung, lässt sich Werkstoff von zwei Seiten in Richtung des Fügepartners (3) umformen. Hierbei entstehen zum einen beidseitig sogenannte Schultern, die das Fügeteil nach dem Prozess axial auf der Welle positionieren, zum anderen entstehen aber auch beidseitig charakteristische Kerben neben der eigentlichen Fügestelle. Diese Kerben führen zunächst zu einer inhärenten Schwächung der Welle und wirken unter Belastung als Sollbruchstellen. Hier ist der Ansatzpunkt für die gezielt eingebrachten Druckeigenspannungen mit dem Ziel die Wirkung der Kerbe ganz oder zumindest teilweise $\mathrm{zu}$ kompensieren und die Lebensdauer des Bauteils unter zyklischer Belastung zu verbessern. Zudem wirkt sich die lokale Verfestigung des Werkstoffs infolge des Kaltwalzprozesses positiv im Hinblick auf die vorhandene Querschnittreduktion aus.

Der eigentliche Fügeprozess lässt sich in Anteile von Form- und Kraftschluss aufteilen. In axialer Lastrichtung wirken die aufgeschobenen Schultern als formschlüssige Fügeverbindung. Weiterhin expandiert die Welle im Bereich zwischen den Walzenpaaren durch das beidseitige Walzen und verspannt den vorher leichtgängig gefügten Fügepartner zu einer kraftschlüssigen Verbindung, die prinzipiell sowohl in axialer als auch in tangentialer Richtung Kräfte und die daraus resultierenden Momente übertragen kann. Die grundsätzliche Machbarkeit, Auslegungskriterien für die Fügeverbindung und eine erste Messung des übertragbaren Drehmoments sind in [15] dargelegt. Der Fokus hier soll im Folgenden auf der Simulation des Fügeprozesses und der resultierenden Eigenspannungsverteilung, sowie der daraus abzuleitenden Eigenschaften hinsichtlich Leichtbaupotentialen und Lebensdauer liegen.

\subsection{Prozessparameter}

Der Radial-Walzprozess findet auf einer modifizierten Schulterwalzmaschine 2-PR-15e der Firma Profiroll statt. Die einstellbaren Prozessparameter sind hierbei der ge- 
Abb. 2 a Diagramm möglicher Walzpfade; b Schematische Ansicht der Walzenzustellung im Prozess

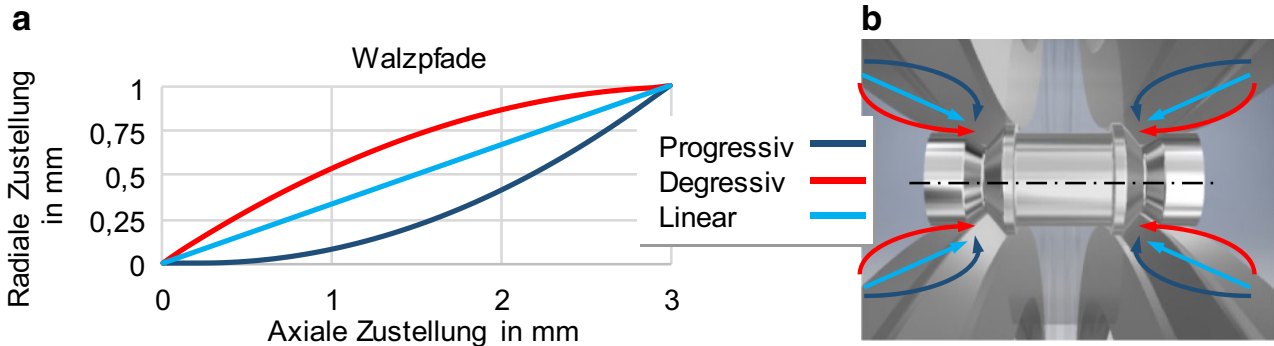

wählte Walzpfad als Kombination aus radialer und axialer Zustellung, siehe auch Abb. 2a, sowie die Drehzahl der Arbeitswalzen, die Vorschubgeschwindigkeit in Form einer wählbaren Prozesszeit sowie eine Kalibrierdauer am Ende des Prozesses, siehe Tab. 1. Weiterhin können die Arbeitswalzen ausgetauscht werden, wodurch sich die Geometrie der Kontaktzone zum Walzgut und die sich ausprägende Form der Kerbe am Ende des Prozesses beeinflussen lässt, Abb. 2b.

Beim Walzpfad lässt sich sowohl die anzufahrende Endzustellung in radialer und axialer Richtung variieren als auch der Weg auf dem diese erreicht wird, siehe progressiver und degressiver Walzpfad in Abb. 2. Bei gleicher angefahrener Endposition lassen sich so die Umformung in Form des Werkstoffflusses und das am Ende entstehende Eigenspannungsprofil verändern, wobei die gefertigte Geometrie annähernd gleichbleibt. Ziel derzeitiger Forschungsarbeiten ist es, diesen Effekt gezielt auszunutzen, um ein vorteilhaftes Eigenspannungsprofil im hochbeanspruchten und gekerbten Querschnitt zu erzeugen während gleichzeitig der Fügeprozess gewährleistet ist.

\subsection{Werkstoffauswahl und Charakterisierung}

Um den Einfluss des Walzprozesses auf die Eigenspannungen im Bereich der entstehenden Fügeverbindung zu untersuchen, liegt der Fokus zunächst auf dem Schaft der entstehenden Fügeverbindung, da sich hier die kritische Kerbe ausbildet, bei der ein Ausgleich der resultierenden Beanspruchungen im Kerbgrund entscheidend für die erreichbare Lebensdauer des Gesamtbauteils ist. Die Entstehung dieser Kerbe lässt sich prozessseitig nicht vermeiden, da hier der Werkstoff umgeformt werden muss, um die Fügeverbindung auszubilden. Die genaue Geometrie und der exakte Radius am Kerbgrund lässt sich zwar mit einer veränderten Walzengeometrie leicht verändern, das generelle Problem bleibt aber weiterhin bestehen.

Tab. 1 Einstellbare Prozessparameter der Walzmaschine

\begin{tabular}{llll}
\hline $\begin{array}{l}\text { Drehzahl Ar- } \\
\text { beitswalzen } \\
\text { in } \mathrm{min}^{-1}\end{array}$ & $\begin{array}{l}\text { Resultierende Dreh- } \\
\text { zahl Walzgut } \\
\text { in } \mathrm{min}^{-1}\end{array}$ & $\begin{array}{l}\text { Prozesszeit } \\
\text { in s }\end{array}$ & $\begin{array}{l}\text { Kalibier- } \\
\text { dauer } \\
\text { in s }\end{array}$ \\
\hline $20-80$ & $\sim 300-1300$ & $2-10$ & $0-5$ \\
\hline
\end{tabular}

Diese Kerbe ist vor allem für die Betriebsfestigkeit unter hohen Lastspielzahlen von großer Bedeutung, da sich ihre Wirkung nicht einfach durch Auswahl eines höherfesten Werkstoffs ausgleichen lässt, wie in [15] ausführlich dargelegt. Dies ist vor allem darin begründet, dass hochfeste Stähle auch eine höhere Kerbempfindlichkeit aufweisen, sodass sich kein Vorteil aus der Substitution ergibt [16]. Zudem zeigen höherfeste Stähle auch eine erhöhte Eigen- und Mittelspannungsempfindlichkeit. Dennoch ist für den hier vorliegenden Einsatzfall ein hochfester Stahl zielführend, da sich umgekehrt auch ein deutlich höheres vorteilhaftes Eigenspannungsniveau im Druckbereich erzeugen lässt. Unter dem Einfluss von Druckmittelspannungen ist eine deutlich stärkere Zunahme der ertragbaren Spannungsamplituden erreichbar als bei weichen Stählen [17]. Für die Untersuchungen wird daher der hochlegierte Stahl 42CrMo4 (1.7225) verwendet, der auch gute Umformeigenschaften in Kaltwalzprozessen zeigt.

Zur Untersuchung eines möglichen Einflusses von kinematischer Verfestigung auf das Werkstoffverhalten des gewählten Werkstoffs bei der Umformung wurden ZugDruck-Versuche durchgeführt. Hierfür wurden kurze Proben mit einem annähernd kubischen Prüfvolumen mit einem zylindrischen Probendurchmesser von $8 \mathrm{~mm}$ und einer parallelen Prüflänge von ebenfalls $8 \mathrm{~mm}$ ausgewählt. Die Versuche wurden auf einer Universalprüfmaschine inspekt $250 \mathrm{kN}$ der Firma Hegewald \& Peschke durchgeführt und mittels eines GOM Aramis 3D 4M Systems aufgezeichnet und ausgewertet. Die Verwendung eines Systems zur digitalen Bildkorrelation hat hierbei den Vorteil, dass neben der Verzerrung des aufgebrachten stochastischen Musters auch der jeweilige effektive Durchmesser der Probe bestimmt werden kann. Die Prüfgeschwindigkeit der Versuche betrug $1 \mathrm{~mm} / \mathrm{min}$ und die Aufnahme der Bilder erfolgte mit einer Frequenz von $10 \mathrm{~Hz}$.

Die Ergebnisse der Versuche mit jeweils unterschiedlicher Vordehnung in Druckrichtung und dann folgender Zugprüfung bis zum Versagen sind in Abb. 3 dargestellt. Im Übergangbereich von elastischer zu plastischer Dehnung bei Lastumkehr ist zunächst eine leichte Verringerung der Fließspannung zu beobachten. Dieser Effekt verschwindet aber bei nur geringerer weiterer Dehnung vollständig und die Fließspannung erreicht wieder das ursprüngliche Ni- 


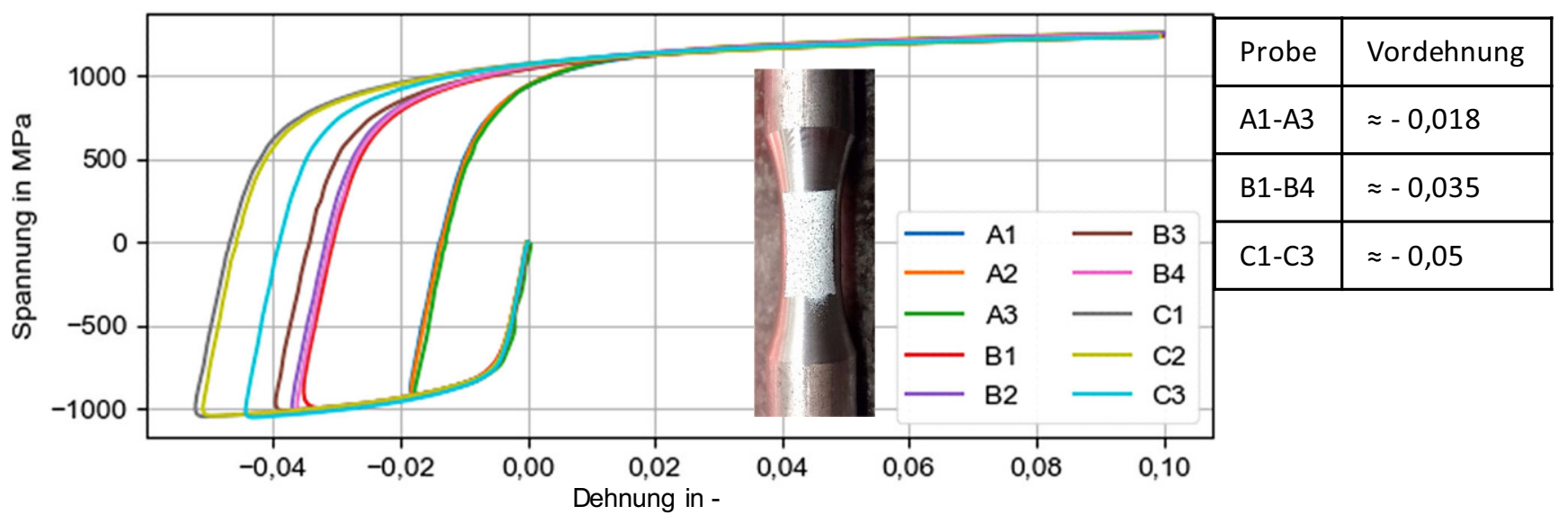

Abb. 3 Ergebnisse der Zug-Druck-Versuche mit unterschiedlichen Vordehnungen in Druckrichtung

Abb. 4 Ergebnisse der Druckversuche und Extrapolationsansätze nach Ghosh und HocketSherby

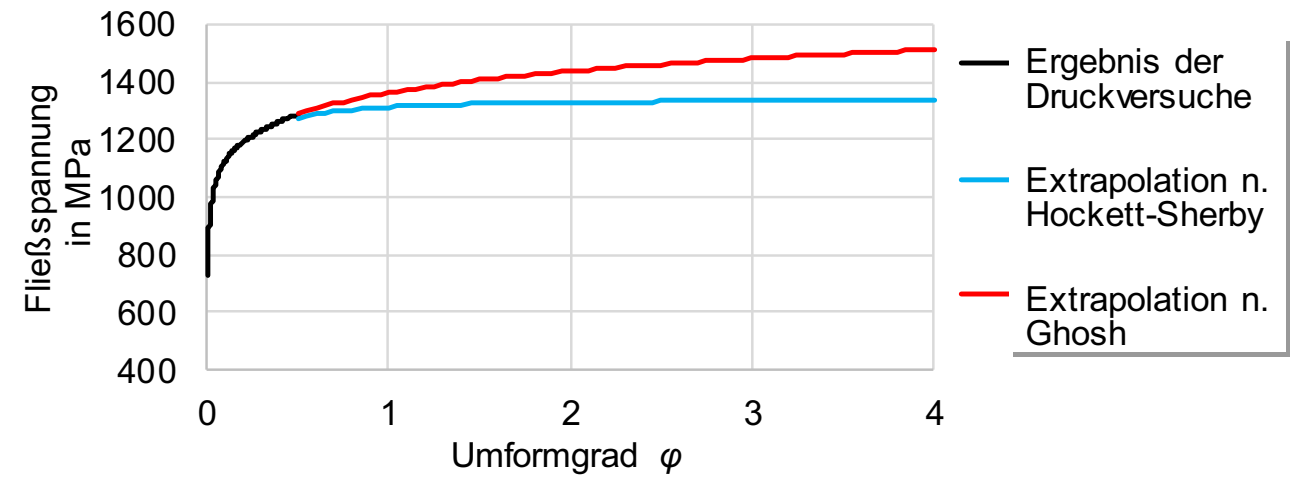

veau, sodass die kinematische Verfestigung im Werkstoffmodell für die Simulation des Walzprozesses vernachlässigt werden kann.

Die Fließkurve für das in den Simulationen verwendete Werkstoffmodell wurde im Druckversuch mit fünf Wiederholungen bis $\mathrm{zu}$ einem Umformgrad von $\varphi=0,5$ ermittelt und durch einen Extrapolationsansatz nach Hocket-Sherby [18] bis $\mathrm{zu}$ einem Umformgrad von $\varphi=4$ erweitert. Wie in Abb. 4 dargestellt, sind die Extrapolationsansätze nach Ghosh [19] und Hocket-Sherby als die beiden möglichen auftretenden Grenzfälle zu betrachten. Der Ansatz nach Hocket-Sherby wurde für die Extrapolation gewählt, da Ghosh im Vergleich zu Eigenspannungsmessungen an Realbauteilen bei der Umformsimulation zu einer großen quantitativen Überschätzung der Eigenspannungen führt.

Auf eine Charakterisierung der Eigenspannungsverteilung im Halbzeug zu Prozessbeginn kann verzichtet werden, da Sensitivitätsanalysen gezeigt haben, dass die hohe lokale Umformung vorher eingeprägte Eigenspannungszustände vollständig überschreibt [20].

\subsection{Modellierung des Walzprozesses}

Das Modell des Walzprozesses wurde mit LS-Prepost v.4.5 erstellt und mit LS-Dyna R11.1.0 unter Verwendung des impliziten Solvers auf dem Hochleistungsrechencluster des Zentrums für Informationsdienste und Hochleistungsrechnen $(\mathrm{ZIH})$ der TU Dresden ausgeführt. Hierbei wurde der Walzprozess in einem vereinfachten $2 \frac{1}{2} \mathrm{D}$-Modell umgesetzt, welches die Rotationsachse des Bauteils als Rotationssymmetrie des Prozessmodells nutzt. Eine weitere Symmetrieebene teilt das Bauteil in axialer Richtung zwischen den gegenüberliegenden Walzenpaaren in der Mitte des Fügepartners, siehe Abb. 5. Auf diese Weise kann die Rechenzeit deutlich verringert werden, was es ermöglicht deutlich schneller Sensitivitätsanalysen prinzipieller Verfahrensvarianten durchzuführen. Aufgrund des gewählten Ansatzes wird der Walzprozess als umlaufende Einschnürung der Welle betrachtet, wobei die im realen Prozess auftretenden punktförmige Belastung des Werkstoffs vernachlässigt wird.

Für das elastisch-plastische Werkstoffmodell der Welle und Nabe wurde eine multi-lineare Annäherung an die Fließkurve des $42 \mathrm{CrMo} 4$ mit isotropen Verfestigungsverhalten gewählt. Um das Verhalten im Kontaktbereich zwischen Walze und Walzgut sowie zwischen Walzgut und Fügepartner abbilden zu können, wird mit einer sehr feinen Vernetzung mit einer Elementgröße von 0,05 mm gearbeitet. Die Reibung im Kontaktbereich folgt dem Coulomb'schen Reibgesetz mit einem Reibzahl $\mu=0,1$. Diese Reibzahl ist 

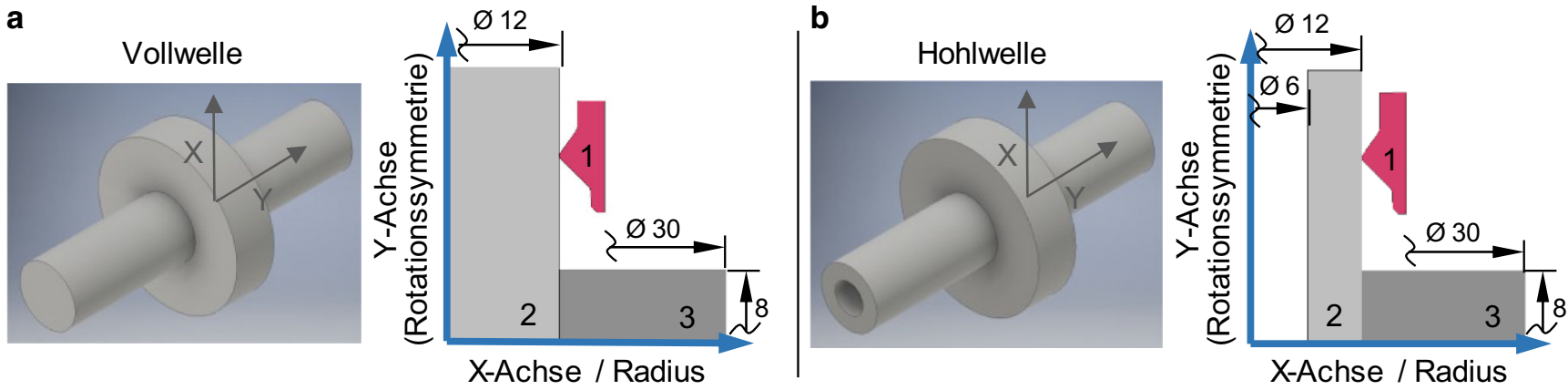

1 - Arbeitswalze

2 - Walzgut / Welle

Abb. 5 Modellaufbau für a Vollwelle und b Hohlwelle mit Y-Achse als rotatorische Symmetrieachse und X-Achse als zusätzliche Symmetrieebene

eine typische Annahme für einen Stahl-Stahl Kontakt mit Mischreibung in der Kaltumformung. Die sehr großen lokalen Deformationen in der Umformzone führen zu einer starken Deformation der Elemente, sodass eine regelmäßige Neuvernetzung notwendig ist. Der Walzprozess wurde hier in vier Varianten vergleichend simuliert; jeweils mit progressivem und degressivem Walzpfad in Kombination mit einer Vollwelle und einer Hohlwelle, siehe Abb. 5.

Der beschriebene vereinfachte Ansatz ist wesentlich schneller als ein vollständiges 3D-Modell - ca. $30 \mathrm{~min}$ Berechnungszeit im SMP-Modus (shared memory parallel processing) gegenüber einigen Tagen mit aktiviertem MMP (massively parallel processing) und einer deutlich höheren Anzahl an Kernen. Trotz der beschriebenen vereinfachenden Annahmen, ist es möglich anhand der Simulationsergebnisse grundsätzliche Betrachtungen bezüglich der resultierenden Eigenspannungszustände in Abhängig der gewählten Walzpfade anzustellen.

Zwar kann es zu Abweichungen im Vergleich zur realen Umformung kommen, besonders hinsichtlich der genauen geometrischen Ausprägung, jedoch lassen sich die relevanten Einflüsse auf die Eigenspannungsverteilung im Kerbgrund dennoch qualitativ und in der Größenordnung sinnvoll untersuchen. Der betrachtete Spannungszustand im gewählten Ansatz weicht vom Spannungszustand eines 3DModells übergreifend $\mathrm{ab}$, jedoch ist die eigentliche Umformzone sehr klein mit einem Radius an der Walzenkante von etwa $0,3 \mathrm{~mm}$. Nur dort wird jeweils die Fließspannung sowohl im Realprozess als auch in der 3D-Simulation in einem sehr kleinen Bauteilvolumen erreicht. Der umgebende Werkstoff verhält sich hingegen elastisch, d.h. vergleichbar mit einem sehr steifen Abstützung. Daraus folgend wirkt ein Druckspannungszustand in Umfangsrichtung welcher dem Spannungszustand im achsensymmetrischen Modell stark ähnelt. Die Hauptspannungen in radialer und tangentialer Richtung in der $2 \frac{1}{2} \mathrm{D}$-Simulation sind direkt voneinander abhängig. In axialer Richtung verhalten sich beide Modelle gleich steif, daher sind die Spannungszustände ähnlich. Die übergreifende Spannungsverteilung zeigt hingegen Abweichungen, was aber aufgrund der sehr lokalisierten Umformzone keinen störenden Einfluss auf die betrachteten lokalen Eigenspannungsprofile im Bereich der Kerbe hat.

\subsection{Ergebnisse der Prozesssimulation}

Bei den Ergebnissen der Simulation mit progressivem und degressivem Walzpfad fällt zunächst auf, dass sich die Kerbgeometrie leicht unterschiedlich ausbildet, sodass sich ein etwas abweichender Kerbfaktor von $K_{\mathrm{t}}=2,7$ für den degressiven Pfad und respektive $K_{\mathrm{t}}=3$,2 für den progressiven ergibt. Die Kerbfaktoren wurde hierfür anhand der Richtlinien von Neuber [21] näherungsweise ermittelt. Dieser Unterschied ist für die nachfolgende Simulation der zu erwartenden Lebensdauer mittels des Örtlichen Konzepts von Bedeutung, siehe Abschn. 3.5. An realen Bauteilen fällt der Unterschied in der Kerbgeometrie zwischen den unterschiedlichen Walzpfaden so gering aus, dass er sich mit einer optischen Vermessung mittels 3D-Scan nicht nachweisen lässt. Der größere Unterschied in den hier gezeigten Simulationsergebnissen ist daher auf die getroffenen Vereinfachungen bezüglich der Rotationssymmetrie zurückzuführen. Die hier diskutierten Ergebnisse dienen einem qualitativen Vergleich unterschiedlicher Walzpfade, da aufgrund der getroffenen Annahmen für die Simulation die absolute Höhe der Eigenspannungen nur als vergleichende Größe dienen können.

Zur Darstellung der Eigenspannungsprofile wird eine Kombination der Vergleichsspannung nach von Mises [22] und der Triaxialität gewählt, da dies eine bessere Beurteilung des vorherrschenden räumlichen Spannungszustands ermöglicht. Die Triaxialität ist hierbei ein Maß für die Mehrachsigkeit eines Spannungszustands und wird als Quotient des hydrostatischen Spannungszustands und der Vergleichsspannung nach von Mises berechnet.

Das Niveau der maximalen Eigenspannungen in den Ergebnissen mit etwa 1150MPa liegt oberhalb der Anfangs- 

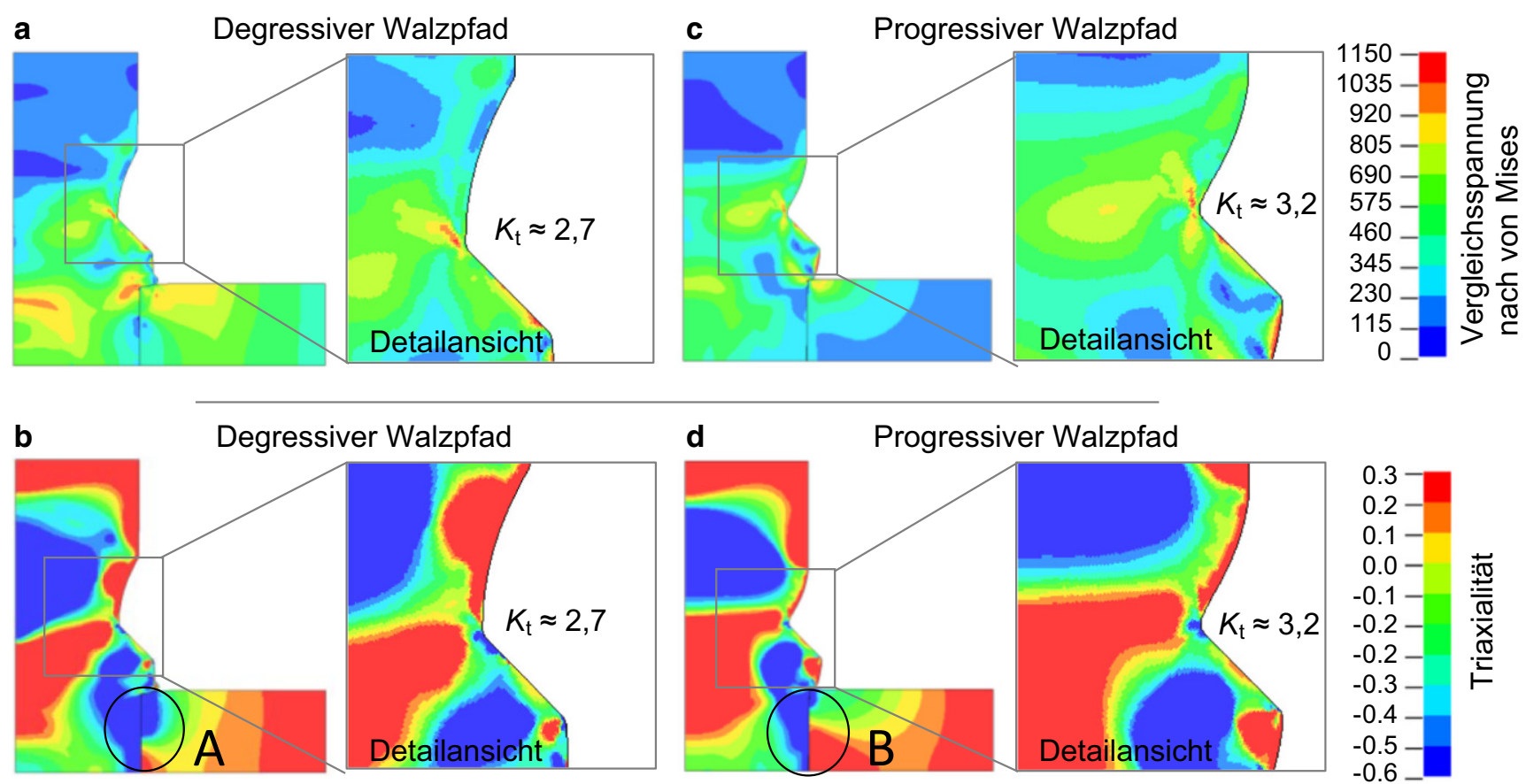

Abb. 6 Ergebnisse der Prozesssimulation für den Fügeprozess mit Vollwelle

fließspannung des Werkstoffs. Diese Zonen weisen einen sehr hohen Umformgrad von bis zu $\varphi=4$ auf, sodass zu diesem Zeitpunkt eine deutlich höhere Fließspannung von etwa $1300 \mathrm{MPa}$ vorliegt, vgl. Abb. 4. Daher sind diese sehr hohen Werte plausibel. Genau im Kerbgrund leicht unterhalb der freien Oberfläche ergeben sich erwartungsgemäß die höchsten Vergleichseigenspannungen, siehe Detailansichten Abb. 6a und Abb. 6c. Die Auswertung der jeweiligen Triaxialität in diesen Bereichen, siehe Abb. $6 \mathrm{~b}$ und Abb. 6d, zeigt, dass es sich um Druckeigenspannungen handelt, wodurch ein positiver Einfluss auf die Betriebsfestigkeit zu erwarten ist. Die Skala für die Triaxialität ist so gewählt, dass Zugeigenspannungen rot und Bereiche mit stärker ausgeprägten Druckeigenspannungen blau dargestellt werden. Gerade der Kerbgrund ist als kritische Zone hinsichtlich einer Rissausbildung zu betrachten, da sich hier Spannungsspitzen ausbilden. Hohe Druckeigenspannungen in dieser Zone sollten daher zu einer deutlichen Verbesserung der Lebensdauer führen. In der Fügezone zwischen Welle und Nabe bildet sich wellenseitig eine Zone vorherrschender Druckeigenspannungen aus, wie an der blauen Zone der Triaxialität deutlich zu erkennen ist. Diese bewirken den Kraftschluss zur umschließenden Nabe. Allerdings wird dieser Bereich zur Nabenmitte hin schmaler, was einem Abfall des dort herrschenden Fugendrucks entspricht, siehe kreisförmige Markierungen $A$ in Abb. 6b und $B$ in Abb. 6d. Dieser Bereich hat also im Sinne der Fügeverbindung einen geringeren Beitrag zur Übertragung von Kräften und Momenten.
Die Auswertung der Ergebnisse der Prozesssimulation mit Hohlwelle zeigt für die Verteilung der Eigenspannungen prinzipiell ein ähnliches Bild. Jedoch sind die Spannungsspitzen im Bereich der Kerbe weniger ausgeprägt, wobei beim progressiven Walzpfad weiterhin eher Druckeigenspannungen dominieren. Beim degressiven Walzpfad hingegen ist im Kerbgrund eher ein Zugspannungszustand vorherrschend. Dies ist nach aktuellem Wissensstand eher ungünstig für die Lebensdauer von Bauteilen. Für die Herstellung gefügter Welle-Nabe-Verbindungen ergibt sich daher ein möglicher Zielkonflikt zwischen einem vorteilhaften Walzpfad im Hinblick auf den Fügeprozess und einem Walzpfad, der zu einer vorteilhaften Eigenspannungsverteilung führt. Besonders interessant im Sinne der Fügeverbindung sind die Fügezonen $C$ in Abb. $7 \mathrm{~b}$ und $D$ in Abb. 7d. Hier bildet sich beim Fügen mit rohrförmiger Welle eine gleichmäßigere Spannungsverteilung über die Breite des Fügespalts aus und die Zone der Druckeigenspannungen ist breiter und reicht bis in die Mitte der Nabe hinein. Dies ist ein Indiz dafür, dass sich beim Fügen mit Hohlwelle günstigere Fügeeigenschaften einstellen lassen.

\subsection{Eigenspannungsmessungen}

Die Messung der Eigenspannungen an realen Bauteilen erfolgte mit einem Prism Bohrlochmesssystem der Firma Stresstech. Dieses System weist die Besonderheit auf, dass keine DMS zum Einsatz kommen, sondern die Verschiebungen auf der Probenoberfläche rund um das Bohrloch durch ESPI (Electronic Speckle Pattern Interferometry) bestimmt 

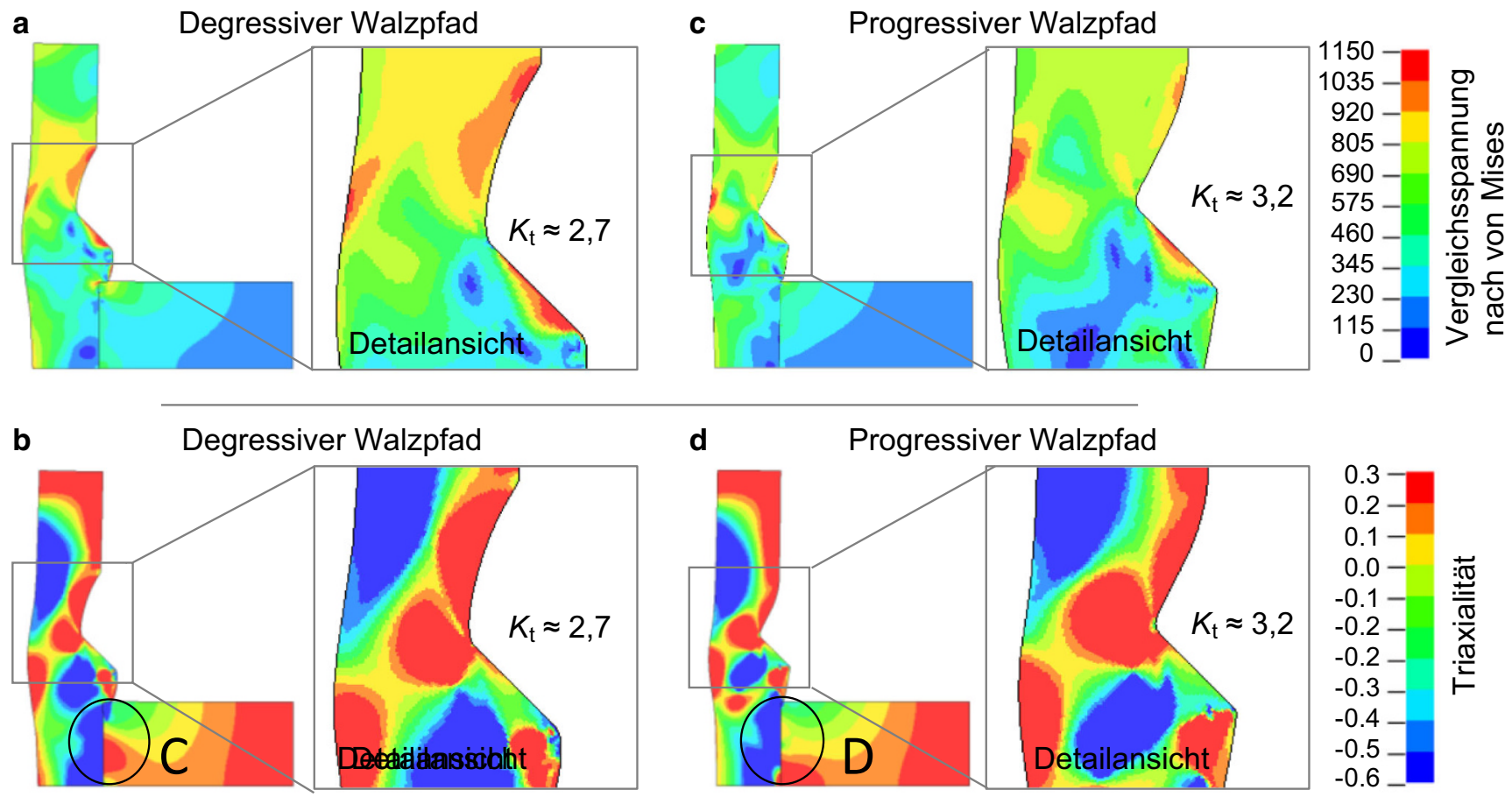

Abb. 7 Ergebnisse der Prozesssimulation für den Fügeprozess mit Hohlwelle

Abb. 8 Messungen der Eigenspannungen vor dem Walzprozess am bearbeiteten Halbzeug

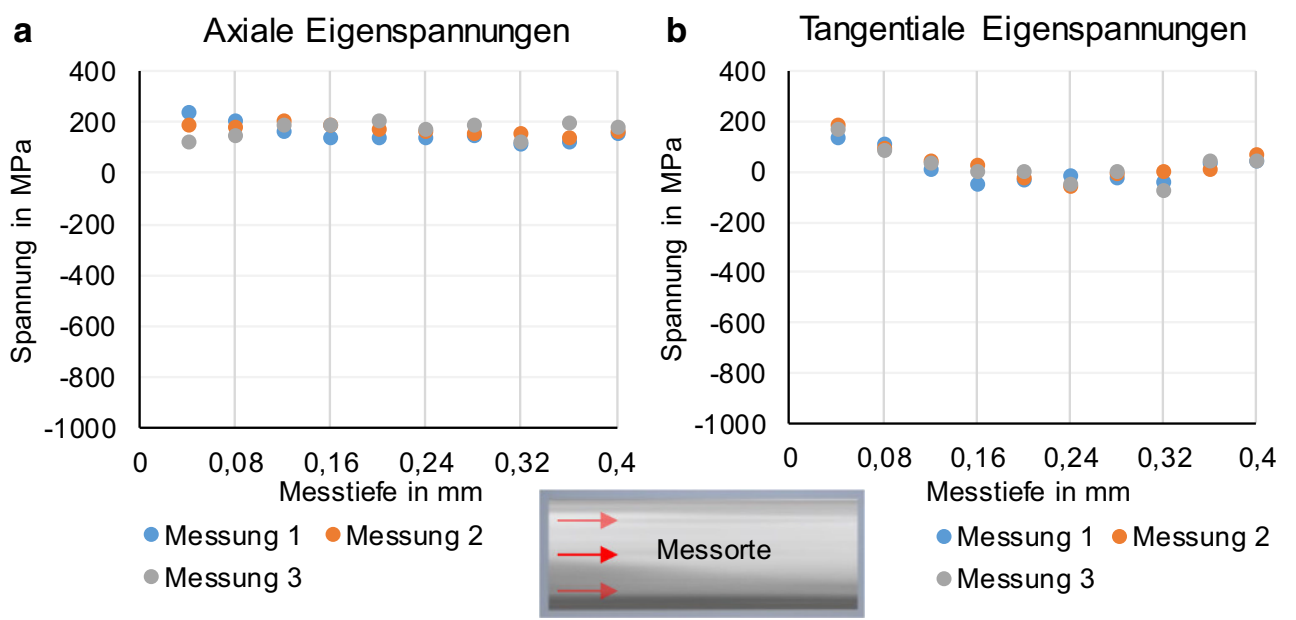

und ausgewertet werden. Zur Probenvorbereitung wurde ein dünner weißer Sprayfilm aufgetragen. Wie bei allen Bohrlochsystemen ist die erreichbare Messtiefe begrenzt, da ab einer gewissen Bohrtiefe die Veränderungen im Eigenspannungszustand im Bauteil nicht mehr zu einer für die Messung benötigten Deformation der Oberfläche führen. Zur Auswertung wird ein ebener Spannungszustand vorausgesetzt und die Tiefenprofile werden mittels eines IntegralAnsatzes von Schajer [23] berechnet.

Die Bohrungen wurden mit einem $0,8 \mathrm{~mm}$ Bohrer in Bohrschritten von jeweils $0,04 \mathrm{~mm}$ bis zu einer Gesamttiefe von $0,4 \mathrm{~mm}$ durchgeführt. In Abb. 8 sind Eigenspannungsmessungen an einem mittels Drehbearbeitung vorbereiteten Halbzeug für den Walzprozess dargestellt. Die drei darge- stellten Messungen erfolgten verteilt auf dem Umfang der gemessenen Probe, siehe auch Abb. 8. Im zweiten Schritt erfolgten dann die Messungen an einer mit degressivem Walzpfad gefertigten Probe. Die Messungen wurden verteilt auf dem Umfang an der Schulter der Kerbe, siehe Detailbild Abb. 9, durchgeführt, da eine Messung genau im Kerbgrund technisch nicht realisierbar ist.

Die Messungen wurden an Vollwellen durchgeführt. In axialer Richtung liegen Zugeigenspannungen von ca. $200 \mathrm{MPa}$ bis zur auswertbaren Tiefe von $0,4 \mathrm{~mm}$ vor. Die tangentialen Zugeigenspannungen zeigen hingegen einen Abfall von ca. 200MPa in der Randzone zu Werten um 0 bei steigender Bohrtiefe. Diese Messungen dienen als 
Abb. 9 Messungen der Eigenspannungen nach dem Walzprozess an der Kerbschulter
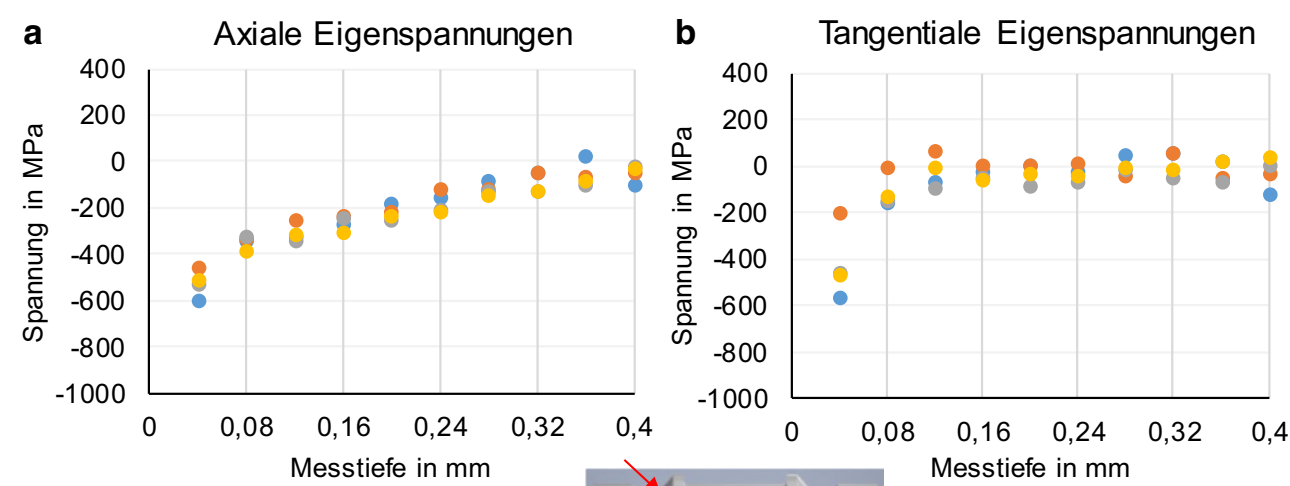

- Messung 1 Messung 2 - Messung 3 Messung 4

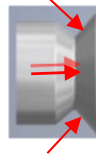

- Messung 1

- Messung 2 Messorte
- Messung 4
Vergleichsgrundlage, um den Einfluss des Walzprozesses auf die Eigenspannungen sichtbar zu machen.

Es ist eine deutliche Veränderung gegenüber dem initialen Eigenspannungsprofil erkennbar. Besonders im oberflächennahen Bereich herrschen deutliche Druckeigenspannungen in der Größenordnung von ca. 600 MPa vor. Dies ist in guter Übereinstimmung mit den Ergebnissen der in Abschn. 3.4 dargestellten Simulationen, da auch hier der Einfluss des Walzprozesses vor allem sehr oberflächennah zu erkennen ist. Quantitativ ist eine direkte Vergleichbarkeit aufgrund der getroffenen Modellvereinfachungen in der Simulation hingegen nicht möglich. Bei steigender Bohrtiefe nähern sich die Eigenspannungen dem Ausgangszustand an, was darauf hindeutet, dass der Einfluss des Walzprozesses in der Tiefe begrenzt ist, was die sehr lokalisierte Umformung nochmals unterstreicht.

\subsection{Lebensdauerbewertung}

Abschließend wird der positive Einfluss der durch den Walzprozess eingebrachten Eigenspannungen auf die Lebensdauer numerisch für Vollwellen gezeigt. Dazu wird die Lebensdauer der progressiv und der degressiv gewalzten Bauteile mithilfe des Örtlichen Konzeptes [24] simuliert.

Das Versagen durch Materialermüdung von duktilen Werkstoffen wird maßgeblich durch den Einfluss des zyklisch elastisch-plastischen Materialverhaltens verursacht. Das Örtliche Konzept ist hierbei ein etabliertes Konzept zur Berechnung der Ermüdungslebensdauer von mechanischen belasteten Bauteilen im Hinblick auf das Versagenskriterium Anrissbildung. In einer linear elastischen FiniteElemente-Simulation wird dabei der kritische Spannungszustand ermittelt und bildet somit die Grundlage für eine elastisch-plastische Abschätzung der Ermüdung mit dem Örtlichen Konzept in einer Post-Processing-Routine. Im Anschluss daran erfolgt die Umbewertung der elastischen Kerbbeanspruchung in eine elastisch-plastische Kerbbe- anspruchung mit Hilfe der Neuber-Umbewertung [21] und eines Hysteresenzählverfahrens [25]. Das zyklische Spannungs-Dehnungs-Verhalten kann mit dem Ansatz von Ramberg-Osgood [26] approximiert werden. Im Nachgang erfolgt die Berechnung eines Schädigungsparameters. Hierfür wurde der Schädigungsparameter von Smith-WatsonTopper [27] gewählt. Der Ermüdungswiderstand kann mit Hilfe der Schädigungsparameter-Wöhlerlinie angegeben werden, die von der experimentell ermittelten Dehnungswöhlerlinie [28] abgeleitet wird. Ein Vergleich des Schädigungsparameters mit der Schädigungsparameter-Wöhlerlinie liefert anschließend die ertragbare Lastwechselzahl, dessen reziproker Wert für den Teilschädigungsbeitrag einer Hysterese steht. Die Akkumulation der Teilschädigung $\mathrm{zu}$ einer Gesamtschädigung erfolgt durch die Miner-Regel [29].

Um die Eigenspannung zu berücksichtigen wird ein initialer Lastwechsel simuliert, dessen Spannungs-DehnungsAntwort bei einmaliger Be- und Entlastung der zu untersuchenden Eigenspannung entspricht Der so entstehende Spannungs-Dehnungs-Pfad bildet die Grundlage für die anschließende Schädigungsrechnung. Als Basis für das initiale Materialverhalten dient die monotone Spannungs-Dehnungs-Kurve.

Auf der Grundlage der in Abschn. 3.4 simulierten Eigenspannungsverteilung und der aus der Belastung in axialer Richtung resultierenden Spannungen wird die theoretische Lebensdauer für jeden Punkt der Welle berechnet. Die höchstbeanspruchte Stelle bestimmt anschließend die Lebensdauer des Gesamtbauteils. Die sich ergebenden Wöhlerlinien für zyklische Zug-/Druck-Belastungen in axialer Richtung sind in Abb. 10 dargestellt. Dabei ist die Anzahl der Lastwechsel bis Anriss $N_{\mathrm{f}}$ über der Nennspannungsamplitude $\sigma_{\mathrm{A}}$ abgebildet. Da die entstehende Kerbe beim progressiven Walzpfad schärfer ist (Kerbformzahl $K_{\mathrm{t}}=3,2$ ) als beim degressiven $\left(K_{\mathrm{t}}=2,7\right)$, entstehen hier geringere Lebensdauern und die Wöhlerkurve liegt niedriger. Zu Ver- 


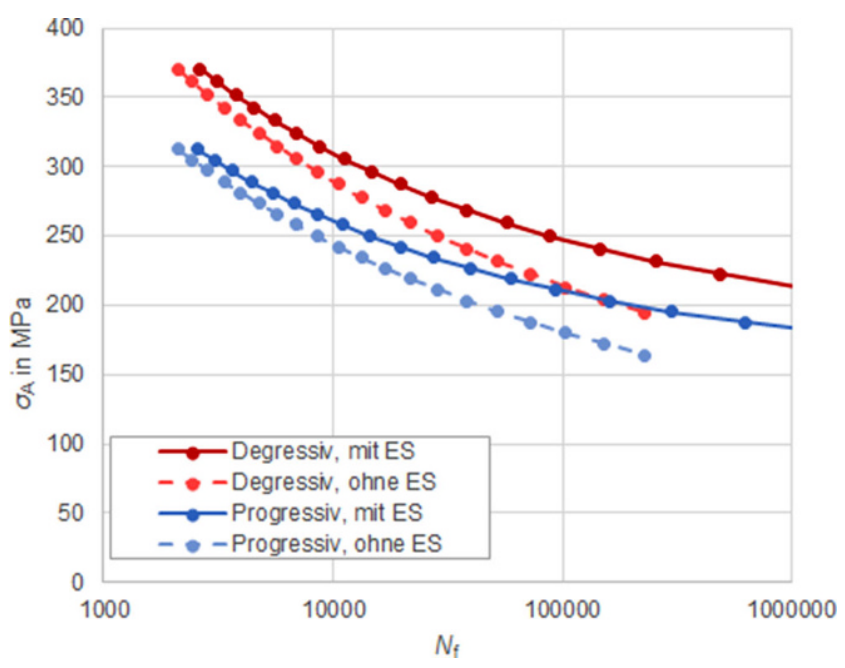

Abb. 10 Wöhlerdiagramm mit Nennspannungsamplitude $\sigma_{\mathrm{A}}$ und Lastwechselzahl bis Anriss $N_{\mathrm{f}}$. Verglichen werden die simulierten Lebensdauern für Bauteile mit progressivem und degressivem Walzpfad mit und ohne Eigenspannungen

gleichszwecken wurden die Simulationen auch für eigenspannungsfreie Bauteile durchgeführt. Es wird ersichtlich, dass die Druckeigenspannungen in der Kerbe bei beiden Prozessvarianten zu einer Verlängerung der Lebensdauer führen.

\section{Fazit}

Der hier vorgestellte Fügeprozess mittels Radial-Walzen ist geeignet, hybride Bauteile form- und kraftschlüssig miteinander zu verbinden. Dies ermöglicht es, die spezifischen Vorteile verschiedener Werkstoffe zu kombinieren und Nutzungs- sowie Leichtbaupotenziale zu erschließen. Die dabei entstehende charakteristische Kerbe in der Wellenkomponente bildet eine Schwachstelle der gefügten Bauteile, da sich dort die Spannungen unter Last konzentrieren und die Ausbildung eines Risses begünstigen. Dieser Herausforderung wird mit einer Kombination der Ausnutzung der Kaltverfestigung des gewählten Werkstoffs (1.7225) und gezielt eingebrachter Druckeigenspannungen in der Kerbe begegnet. Besonders die Druckeigenspannungen wirken hierbei verlängernd auf die Lebensdauer. Sie lassen sich gezielt über eine günstige Wahl des Walzpfads zur Herstellung der Fügeverbindung beeinflussen, wie sowohl in numerischen Analysen als auch durch Eigenspannungsmessungen an Realbauteilen gezeigt wird. Es können sehr hohe Eigenspannungen in der kritischen Zone des Kerbgrunds eingebracht werden, die einen deutlichen Einfluss auf die Lebensdauer haben, wie auch die Lebensdauerberechnungen mittels des Örtlichen Konzepts klar belegen.
Danksagung Die Autoren bedanken sich bei der Deutschen Forschungsgemeinschaft (DFG) für die finanzielle Unterstützung der Forschungsarbeit im Rahmen des Schwerpunktprogramms „SPP2013“ unter den Fördernummern BR 3500/21-2 und KA 3309/7-2.

Funding Open Access funding enabled and organized by Projekt DEAL.

Interessenkonflikt C. Guilleaume, D. Kühne, M. Kästner und A. Brosius geben an, dass kein Interessenkonflikt besteht.

Open Access Dieser Artikel wird unter der Creative Commons Namensnennung 4.0 International Lizenz veröffentlicht, welche die Nutzung, Vervielfältigung, Bearbeitung, Verbreitung und Wiedergabe in jeglichem Medium und Format erlaubt, sofern Sie den/die ursprünglichen Autor(en) und die Quelle ordnungsgemäß nennen, einen Link zur Creative Commons Lizenz beifügen und angeben, ob Änderungen vorgenommen wurden.

Die in diesem Artikel enthaltenen Bilder und sonstiges Drittmaterial unterliegen ebenfalls der genannten Creative Commons Lizenz, sofern sich aus der Abbildungslegende nichts anderes ergibt. Sofern das betreffende Material nicht unter der genannten Creative Commons Lizenz steht und die betreffende Handlung nicht nach gesetzlichen Vorschriften erlaubt ist, ist für die oben aufgeführten Weiterverwendungen des Materials die Einwilligung des jeweiligen Rechteinhabers einzuholen.

Weitere Details zur Lizenz entnehmen Sie bitte der Lizenzinformation auf http://creativecommons.org/licenses/by/4.0/deed.de.

\section{Literatur}

1. Yang DY, Bambach M, Cao J, Duflou JR, Groche P, Kuboki T, Sterzing A, Tekkaya AE, Lee CW (2018) Flexibility in metal forming. Ann Cirp 67(2):743-765

2. Groche P, Fritsche D, Tekkaya AE, Allwood JM, Hirt G, Neugebauer R (2007) Incremental bulk metal forming. Ann Cirp 56(2):635-656

3. Mori K-I, Bay N, Fratini L, Micari F, Tekkaya AE (2013) Joining by plastic deformation. Ann Cirp 62(2):673-694

4. James MN, Hughes DJ, Chen Z, Lombard H, Hattingh DG, Asquith D, Yates JR, Webster PJ (2007) Residual stresses and fatigue performance. Eng Fail Anal 14(2):384-395

5. Volk W, Vogt S, Stahl J, Prauser S (2019) Introduction to residual stresses in production technology. Prod Eng Res Devel 13:119-121

6. Pavliuchenko P, Hirt G, Teller M (2021) Analysis and manufacturing of bistable metallic profiles. Arch Appl Mech. https://doi.org/ 10.1007/s00419-021-01916-2

7. Vogt S, Neuwirth T, Schauerte B, Weiss HA, Falger PM, Gustschin A, Schulz M, Hameyer K, Volk W (2019) Extend of embossing-related residual stress on the magnetic properties evaluated using neutron grating interferometry and single sheet test. Prod Eng Res Devel 13:211-217

8. Fallahiarezoodar A, Gupta T, Goertemiller C, Altan T (2019) Residual stresses and springback reduction in U-channel drawing of A15182 by using a servo press and servo hydraulic cushion. Prod Eng Res Devel 13:219-226

9. Franceschi A, Stahl J, Kock C, Selbmann R, Ortmann-Ishkina S, Jobst A, Merklein M, Kuhfuß B, Bergmann M, Behrens B-A, Volk W, Groche P (2021) Strategies for residual stress adjustment in bulk metal forming. Arch Appl Mech. https://doi.org/10.1007/ s00419-021-01903-7

10. Radaj D, Vormwald M (2007) Ermüdungsfestigkeit, 3. Aufl. Springer, Berlin Heidelberg 
11. Franceschi A, Kaffenberger M, Schork B, Hoche H, Oechsner M, Groche P (2019) Observations on the stability of the residual stresses after cold forming and unidirectinal loading. Prod Eng Res Devel 13:157-167

12. Coffin LFJ, Schenectady NY (1954) A study of the effects of cyclic thermal stresses on a ductile metal. Trans Am Soc Mech Eng 76:931-950

13. Delgado P, Cuesta II, Alegre JM, Daz A (2016) State of the art of deep rolling. Precis Eng 46:1-10

14. Blasón S, Rodríguez C, Belzunce J, Suárez C (2017) Fatigue behaviour improvement on notched specimens of two different steels through deep rolling, a surface cold treatment. Theor Appl Fract Mec 92:223-228

15. Brosius A, Guilleaume C (2020) New cross-rolling process for joining of hybrid components. Ann Cirp 69(1):245-248

16. Macherauch EH, Wohlfart H (1985) Eigenspannungen und Ermüdung. In: Munz D (Hrsg) Ermüdungsverhalten metallischer Werkstoffe. DGM-Informationsgesellschaft, Oberursel

17. Sonsino MC (2009) Light-weight design chances using highstrength steels. Mat-wiss u Werkstofftech 38(1):9-22

18. Hockett JE, Sherby OD (1975) Large strain deformation of polycrystalline metals at low homologous temperatures. J Mech Phys Solids 23:87-98

19. Ghosh A (1977) Tensile instability and necking in materials with strain hardening and strain-rate hardening. Acta Met 25(12):14131424
20. Guilleaume C, Brosius A (2019) Simulation methods for skew rolling. Procedia Manuf 27:1-6

21. Neuber H (1961) Theory of stress concentration for shear-strained prismatical bodies with arbitrary nonlinear stress-strain law. J Appl Mech 28(4):544-550

22. v. Mises R (1913) Mechanik der festen Körper im plastisch-deformablen Zustand. Nachrichten Von Ges Wissenschaften Zu Göttingen Math Kl 1913:582-592

23. Schajer GS, Rickert TJ (2011) Incremental computation technique for residual stress calculations using the integral method. Exp Mech 51(7):1217-1222

24. Kühne D, Guilleaume C, Seiler M, Hantschke P, Ellmer F, Linse T, Brosius A, Kästner M (2019) Fatigue analysis of rolled components considering transient cyclic material behaviour and residual stresses. Prod Eng Res Devel 13:189-200

25. Clormann UH, Seeger T (1986) Rainflow-HCM - Ein Zählverfahren für Betriebsfestigkeit auf werkstoffmechanischer Grundlage. Stahlbau 55(3):65-71

26. W. Ramberg, W.R. Osgood: Description of stress-strain curves by three parameters. NACA Technical note No. 902, 1943.

27. Smith KN, Watson P, Topper TH (1970) A stress-strain function for the fatigue of metals. J Mater 5(4):767-778

28. Manson S (1965) Fatigue: a complex subject—some simple approximations. Exp Mech 5(4): 193-226

29. Miner MA (1945) Cumulative keydamage in fatigue. J Appl Mech 12(3):321-332 